\title{
Techniques for three-dimensional displacement vector using ground-based interferometric synthetic aperture radar
}

\author{
L Leoni IDS Ingegneria Dei Sistemi, Italy \\ G Spencer IDS Australasia, Australia \\ N Coli IDS Ingegneria Dei Sistemi, Italy \\ F Coppi IDS Ingegneria Dei Sistemi, Italy
}

A Michelini IDS Ingegneria Dei Sistemi, Italy

\begin{abstract}
Over the past 10 years slope monitoring radar via the interferometry technique has evolved as an important tool for safety critical monitoring of pit wall movements in surface mining. Whilst radar can provide near real-time high spatial resolution surface displacement movement, the displacement vector measured using interferometry is line-of-site (LOS) to the radar position and does not provide a spatially oriented vector.

Using two or more ground-based interferometric synthetic aperture radar (GBInSAR) systems to rapidly record continuous spatially dense LOS displacement data of an open pit slope in 'stereo', has potential to provide detailed vector movement across an entire wall or whole pit. The radar displacement vectors can be combined to create a pseudo three-dimensional (3D) displacement map of the pit slope using millions of monitoring points. Data collected from several radar systems has potential to greatly improve the understanding of the 3D kinematics of a large rock slope and further assist in better mine planning and design thus allowing more efficient mine operations. This paper will cover examples and analysis of pseudo $3 D$ displacement map data from GBInSAR systems.
\end{abstract}

\section{Introduction}

Ground-based interferometric radar (GBInRad) systems are a class of terrestrial remote sensing imaging systems, based on radar interferometric techniques (Monserrat et al. 2014; Caduff et al. 2015).

Interferometric radar imaging systems operate by emitting a phase coherent signal in the microwave frequency band and then receiving the reflected arrival of the signal from the targets of interest. The basic principle of interferometry involves the comparison of two images, where the phase image from one measurement is subtracted from another one. The principal application of GBInRad systems is deformation monitoring, since the phase difference between the two radar images can be related to the displacement that occurred with respect to the line-of-site (LOS), (Tarchi 1999, 2003; Reeves 2000; Luzi 2010; Farina et al. 2011).

The main advantages of GBInRad systems compared to other deformation measurement techniques are:

- Remote sensing.

- High sensitivity to small deformations (up to $0.1 \mathrm{~mm}$ ).

- Long range of measurements (up to $5 \mathrm{~km}$ ).

- Imaging capability which allows the system to simultaneously perform a vast number of measurements (up to 300,000 pixel).

- Fast scan time (up to a few seconds). 
The main limitation of all GBInRad systems is their capability of only detecting deformations along the LOS of the sensor, although actual wall movement may deform in any direction in space. This represents a limitation of the technique with respect to being able to estimate the full three-dimensional (3D) deformation vector.

Excluding integration between GBInRad and other measurement systems (Yue et al. 2015) and considering only standard GBInRad systems, it is therefore necessary to apply some measurement strategy, or some geometrical assumptions, on the deformation direction in order to try to reconstruct the actual 3D displacement vector.

If the deformation direction is known the measured LOS deformation can be back-projected onto a modelled $3 \mathrm{D}$ total deformation rate. Alternatively, if the displacement direction is not well understood, by combining interferometric displacements measured from different positions in space, it is possible to reconstruct the pseudo 3D displacement vector of targets (Giunta et al. 2013; Zeng et al. 2015).

With this approach, for spaceborne radar systems, it was possible to reconstruct the 3D displacement field from glaciers working on the ascending and descending passes of satellites (Mohr et al. 1998). A similar concept can be applied to GBInRad, where at least two different measurement positions and basic assumptions of the displacement mechanics can be combined to infer a 3D displacement pattern (Severin et al. 2011, 2014; Salvoni et al. 2015).

This paper explains how it was possible to develop software capable of automatically reconstructing the pseudo 3D displacement vector using multiple radars, while at the same time integrating information acquired by total stations using prisms, and how these tools, when used together, can bring great benefits in understanding the kinematics and dynamics of pit-slope displacements.

\section{Radar-target geometry}

When considering a moving target within the area monitored by radar, each measurement acquired by the system will represent just a component of the real displacement vector. This component will be the projection of the real displacement vector over the LOS of the radar, that is, the line between the monitoring radar and the moving target. Assuming that two radars are covering the same moving target but with different LOS (radars are installed on different sectors of the pit), it is then possible to calculate the direction on the horizontal plane of the real displacement vector as shown in Figure 1.

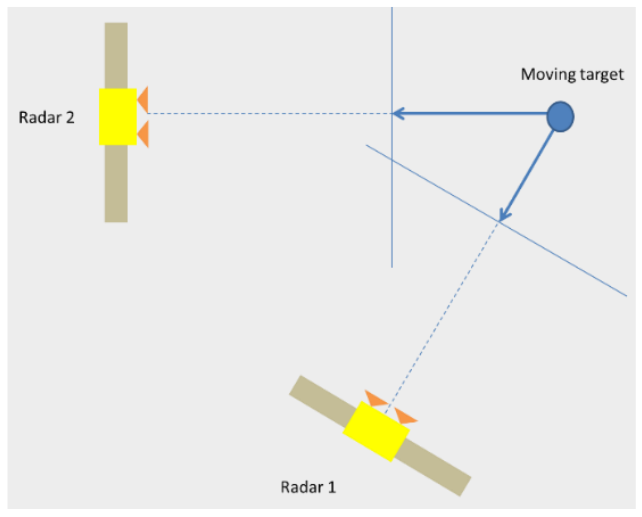

(a)

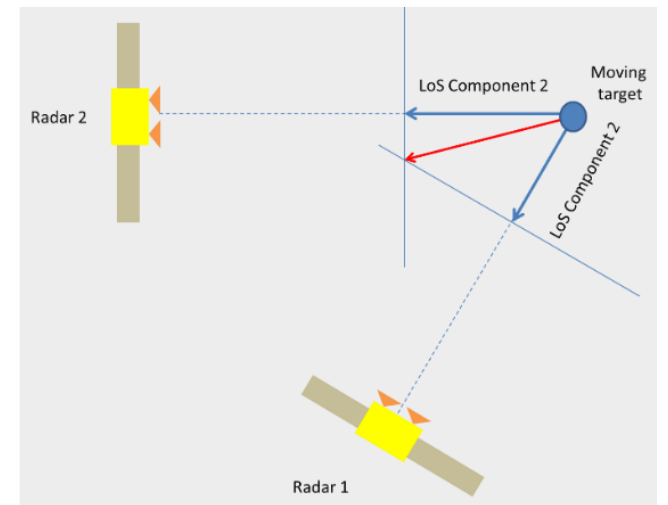

(b)

Figure 1 (a) Radar system geometry with two radars and one moving target. Measurement of different components from different LOS; and, (b) calculus of the direction of the real displacement vector direction on the horizontal plane

With only two units measuring, using two different LOS, it is only possible to obtain the direction of the real displacement vector on the horizontal plane (assuming that the radars are installed at similar elevations). This is because we do not have any information on the vertical inclination of the displacement vector (Figure 2). 


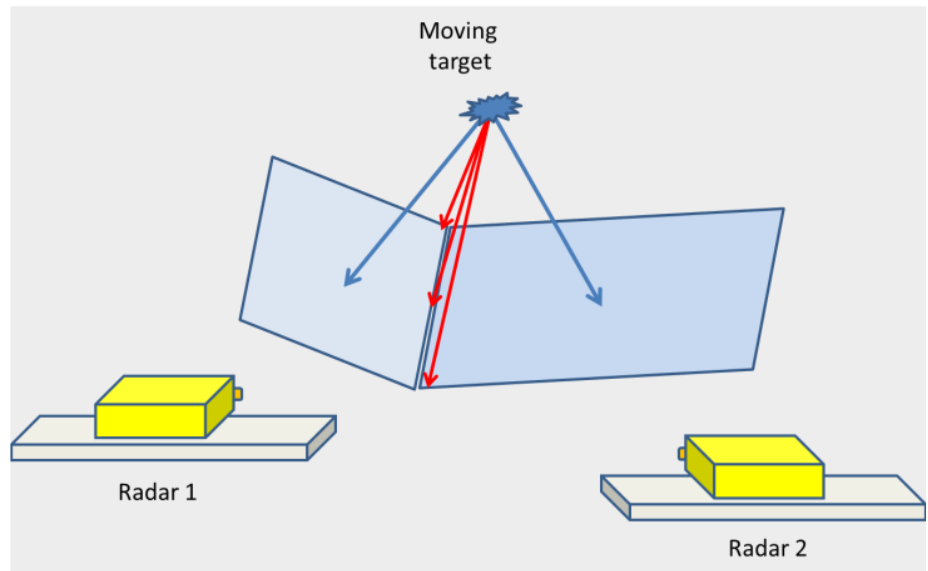

Figure 2 Radar system geometry with two radars and one moving target. Measuring two different components from different LOS will not resolve the entire geometrical triangulation, providing instead just the direction on the horizontal plane (if the two radars are installed at similar elevations)

With a three radar network monitoring configuration, using three different LOS, it will be possible to reconstruct the real displacement vectors by considering the three different components measured by the radars (Figure 3).

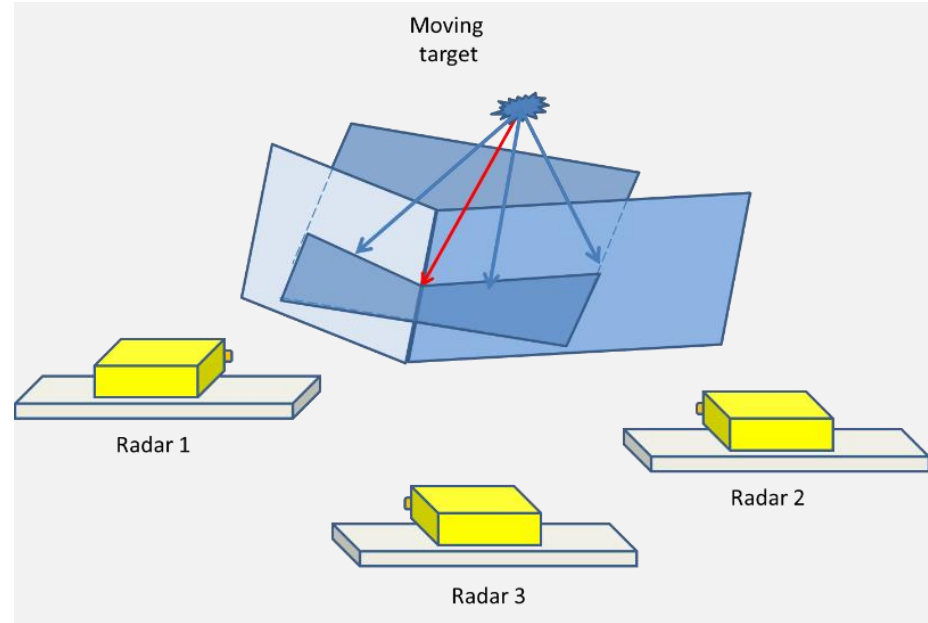

Figure 3 Three radar network and one moving target. Measuring three different components from different LOS will resolve the geometrical triangulation, providing the direction and magnitude of real displacement vectors

In the case of three radars covering the same area, the equation that describes how each different component relates to the real displacement vector can be summarised as:

$$
\left[\begin{array}{l}
d_{P_{1}} \\
d_{P_{2}} \\
d_{P_{3}}
\end{array}\right]=\left[\begin{array}{lll}
u_{P_{1} x} & u_{P_{1} y} & u_{P_{1} z} \\
u_{P_{2} x} & u_{P_{2} y} & u_{P_{2} z} \\
u_{P_{3} x} & u_{P_{3} y} & u_{P_{3} z}
\end{array}\right] \cdot\left[\begin{array}{l}
d_{P x} \\
d_{P y} \\
d_{P z}
\end{array}\right]
$$

where: $d_{P_{1}}, d_{P_{2}}, d_{P_{3}}$ represents the different components measured from the LOS of each different radar and $d_{P_{x}}, d_{P_{y}}, d_{P_{z}}$ are the components of the real displacement vector with respect to each axis, as shown in Figure 4. 


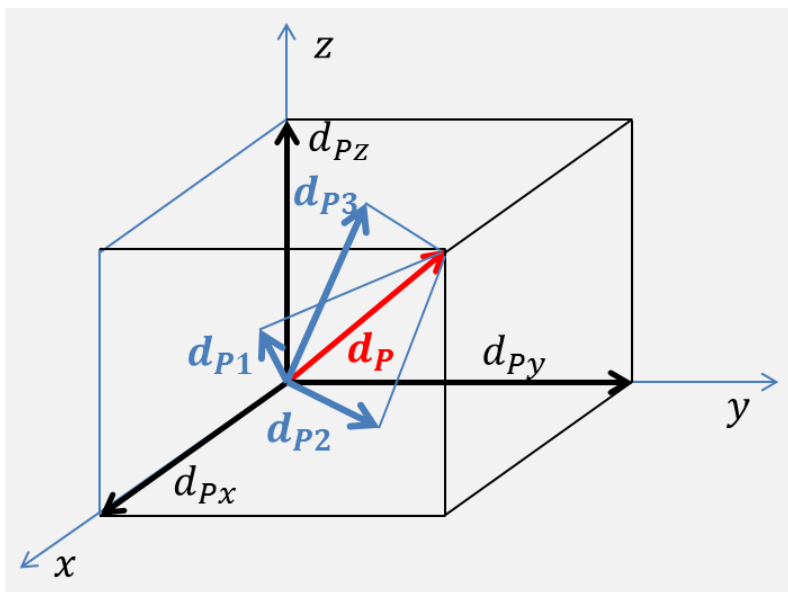

Figure 4 Decomposition of a real displacement vector. Dp1, dp2, dp3 are the LOS components for each radar, with dp the resultant

\section{Radar data integration and pseudo 3D displacement vector}

In case of multiple radar units, real aperture radars (RAR) typically operate independently and separately display and analyse RAR data subsets within the open pit mining area. Software developed for synthetic aperture radar (SAR) technology (Farina el al. 2011, 2012) allows data acquired by multiple SAR systems to be integrated on a single monitoring platform. The full pit radar monitoring software is called Guardian FPM360 which is designed to be used with the commercial SAR system Image by Interferometry Survey (IBIS).

With full pit monitoring, all the data acquired by different SAR radars are shown on one display interface where data can be singularly managed and analysed. With the high spatial resolution of SAR, at long distance monitoring range, it is possible to achieve a full pit monitoring coverage and still be able to critically monitor sub-bench to multi-bench failures. The early recognition of both large scale and bench scale instability over all pit walls, without the need of prior knowledge of the moving areas (as may happen with short range radar), allows an increase in the movement awareness and understanding of the slope behaviour.

The concept of the Guardian FPM360 full pit monitoring, based on the capabilities of IBIS radar, brings the following benefits:

- $24 / 7$ safety-critical and background monitoring over the entire pit for full situation awareness on impending and potential failures.

- Optimisation of conventional monitoring system installations, like prisms for total stations or extensometers, by detecting new activations in previously-stable areas.

- Optimisation of production planning by developing proper mitigation strategies according to the long-term information provided by the background monitoring (e.g. dewatering, optimised blasting techniques etc.).

IBIS's high spatial resolution and wide coverage allows optimal full pit monitoring using minimal number of systems. Depending on the pit geometry and mine operational requirements, typically 2-4 systems covers a large full pit area; noting, historically, in a typical operational configuration of IBIS systems they are positioned in the pit to maximise area coverage and have minimal monitoring area overlap between each system. As a result, previously designed full pit monitoring currently being used in open pit mines are effectively configured for 1D LOS vectors, to be generated, due to minimal area overlap.

With this new capability, of being able to calculate LOS vectors in areas of overlap between SAR radar systems, the configuration for positioning, and the number of radar systems required in the pit, will be based on what hazard areas need multi-dimensional deformation vector monitoring, whether 2 or 3D are needed and how much area overlap is needed across radar sensors. 
The area of interest can be covered by two or more radars installed in different positions in the pit to optimise the overlap coverage area. This will allow the targeted deformation to be measured from different LOS of each radar system deployed. In these cases for each overlapping pixel covered by two radars, it will be possible to calculate the $2 \mathrm{D}$ vector or with three radars, a $3 \mathrm{D}$ displacement vector. The two radar showing the 2D vector movements is shown in Figure 5.

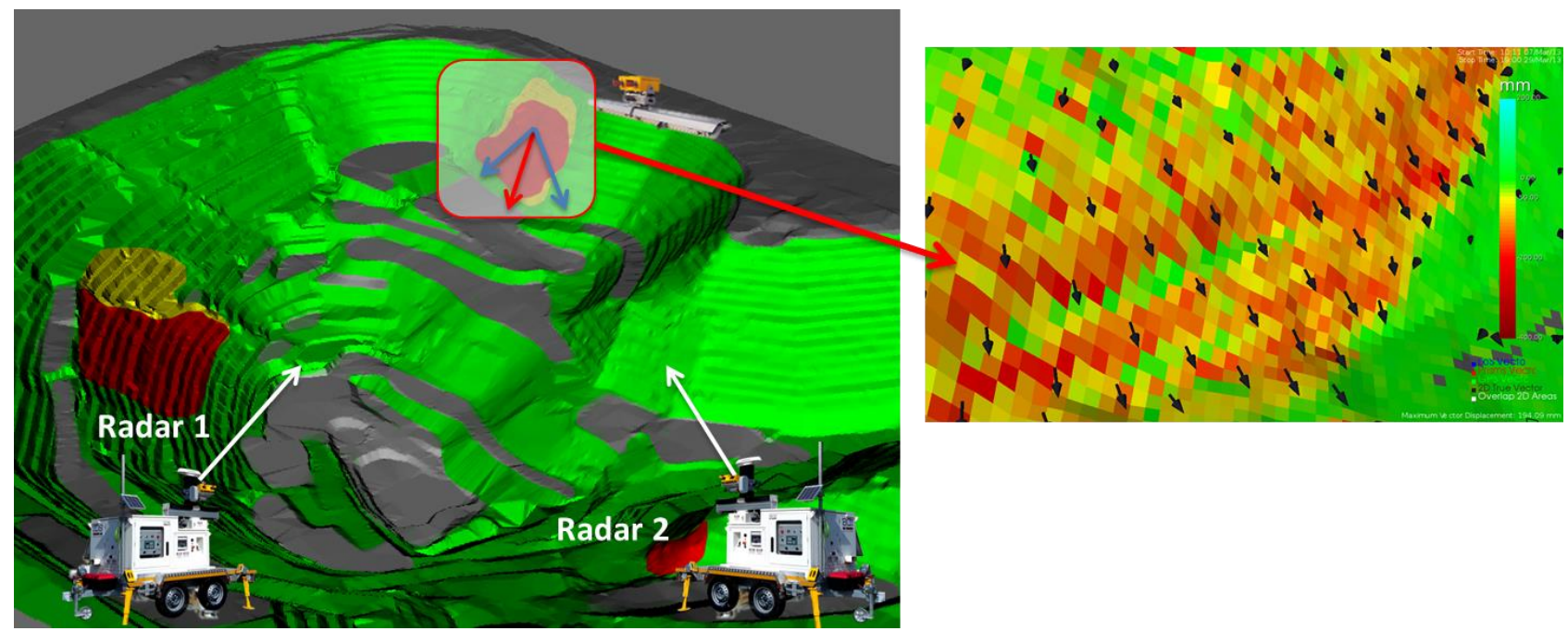

Figure 5 Output of the real displacement vector calculation obtained with two IBIS radars

There are several types of radar configurations that can be implemented to optimise multi-dimensional deformation vector monitoring and maintain broad area coverage. For example, combining 2-4 long-range IBIS monitoring radars, at semi-permanent locations around the pit (to provide full area coverage), with a mobile, short-range and wide coverage IBIS radar, potentially allows 2 or 3D deformation vector monitoring at targeted areas of concern. With this configuration, it is possible to still achieve a broad area coverage and reasonable area of overlap using a minimal number of systems.

Some benefits of detecting real-time movement directions and magnitudes are:

- Support for better understanding the failure mechanism for ongoing complex deformations, both at the slope scale and at the bench scale, thanks to the high resolution, even at long ranges.

- Mapping the evolution of the movement according to the geo-structural setting, including areas with complicated structures and/or varying material types and strengths. High resolution vector movement could potentially be very useful in assisting the understanding of more subtle, smaller areas of movement in brittle material deformations.

- Exploiting the extreme accuracy, fast scan time and high resolution of SAR technology with lower LOS measurement limitations.

- Planning adequate mitigation strategies and emergency action plans according to the measured runout movement direction.

- More flexibility to focus on critical areas.

A good challenge, which occurs when using SAR technology in Guardian FPM360 mode, is being able to analyse, in detail, all of the small localised movement found in the high spatial resolution. This is particularly the case when new IBIS systems are initially established in open pits, where the new additional data is showing many small areas of movement not previously known. Viewing and analysing spatially dense vector data, across the whole pit, would also be a good challenge in identifying information not previously known.

In addition to being able to exploit the direction and magnitude of movement, overlap of radar image areas will also provide another form of data redundancy for quality checks and measurements resulting in even 
higher degrees of confidence in the data, especially in those areas where one of the radar is experiencing a lower signal to noise ratio.

To provide additional redundancy self-checking, a natural extension to near real acquisition and viewing of the radar displacement vector data is to also integrate, cross reference and analyse movement differences in near real-time with other sources of vector displacement data such as automated prism monitoring data (Salvoni et al. 2015). Depending on the number of radar systems used to obtain radar vector data, this approach would need to make allowances for LOS adjustments between relative radar displacement vector data and prism data.

Integration of prisms measurements have been already included in Guardian FPM360, with real-time measurement information, from total station, being shared and automatically imported at server level between the radar monitoring platform and the native prisms database, from different vendors.

\section{Conclusion}

This paper provides an approach for using multiple SAR systems to obtain whole of pit surface vector displacement that is acquired in near real-time (rapid temporal) and high spatial resolution. The benefit of using this approach, especially in conjunction with automated prism monitoring, is the capture of detailed vector data that will greatly assist in understanding the full 3D kinematics, especially in complex geo-structural mining operations.

\section{References}

Caduff, R, Schlunegger, F, Kos, A \& Wiesmann, A 2015, 'A review of terrestrial radar interferometry for measuring surface change in the geosciences', Earth Surface Processes and Landforms, vol. 40, no. 2, pp. 208-228.

Farina, P, Leoni, L, Babboni, F, Coppi, F, Mayer, L \& Ricci, P 2011, 'IBIS-M, Innovative Radar for Monitoring Slopes in Open-Pit Mines', in Slope Stability 2011, International Symposium on Rock Slope Stability in Open Pit Mining and Civil Engineering, Vancouver, Canada.

Farina, P, Leoni, L, Babboni, F, Coppi, F, Mayer, L, Coli, N \& Helbawi, A 2012, 'Monitoring open pit slopes through Slope Monitoring Radar based on Synthetic Aperture Radar', in Proceedings RockEng 2012 21st Canadian Rock Mechanics Symposium, Edmonton, Canada, p. 6.

Giunta, G, Guarnieri, AM, D’Aria, D, Falcone, P, Speziali, F \& Maggi, L 2013, 'A Radar-Based System to Estimate the 3D Vibrational Motion of Gas Pipes', 2013 14th International Radar Symposium (IRS), IEEE, vol. 2, pp. 871-876.

Luzi, G 2010, 'Ground based SAR interferometry: a novel tool for Geoscience', INTECH Open Access Publisher.

Mohr, JJ, Reeh, J \& Madsen, SN 1998, 'Three-dimensional glacial flow and surface elevation measured with radar interferometry', Nature, vol. 391, pp. 273-276.

Monserrat, O, Crosetto, M \& Luzi, G 2014, 'A review of ground-based SAR interferometry for deformation measurement', ISPRS Journal of Photogrammetry and Remote Sensing, vol. 93, pp. 40-48.

Reeves, BA, Stickley, GF, Noon, D \& Longstaff, ID 2000, 'Developments in monitoring mine slope stability using radar interferometry', in Proceedings of Geoscience and Remote Sensing Symposium (IGARSS), vol. 5, pp. 2325-2327.

Salvoni, M, Abbot, K \& Dight, PM 2015, 'Improvement of pseudo-3D pit displacement mapping technique through geodetic prism data integration', in PM Dight (ed.), Proceedings of the Ninth Symposium on Field Measurements in Geomechanics, Australian Centre for Geomechanics, Perth, pp. 185-194.

Severin, J, Eberhardt, E, Leoni, L \& Fortin, S 2011, 'Use of ground-based synthetic aperture radar to investigate complex 3-D pit slope kinematics', in Proceedings of Slope Stability 2011: International Symposium on Rock Slope Stability in Open Pit Mining and Civil Engineering, Vancouver.

Severin, J, Eberhardt, E, Leoni, L \& Fortin, S 2014, 'Development and application of a pseudo-3D pit slope displacement map derived from ground-based radar', Engineering Geology, vol. 181, pp. 202-211.

Tarchi, D, Rudolf, H, Luzi, G, Chiarantini, L, Coppo, P \& Siber, AJ 1999, 'SAR interferometry for structural changes detection: a demonstration test on a dam', in Proceedings of the Geoscience and Remote Sensing Symposium, vol. 3, pp. 1522-1524.

Tarchi, D, Casagli, N, Fanti, R, Leva, DD, Luzi, G, Pasuto, A, Peiraccini, M \& Silvano, S 2003, 'Landslide monitoring by using ground-based SAR interferometry: an example of application to the Tessina landslide in Italy', Engineering Geology, vol. 68, pp. 15-30.

Yue, J, Yue, S, Wang, X \& Guo, L 2015, 'Research on multi-source data integration and the extraction of three-dimensional displacement field based on GBSAR', International Workshop on Thin Films for Electronics, Electro-Optics, Energy and Sensors, International Society for Optics and Photonics.

Zeng, T, Mao, C, Hu, C, Yang, X \& Tian, W 2015, 'Multi-static MIMO-SAR three dimensional deformation measurement system,' in Proceedings of Synthetic Aperture Radar (APSAR), Singapore, IEEE, pp. 297-301. 